\title{
Suppressive Effect of Orthovanadate on Hepatic Stellate Cell Activation and Liver Fibrosis in Rats
}

\author{
Yuji Nishikawa, ${ }^{*}$ Naoto Ohi, ${ }^{*}$ Akiko Yagisawa, ${ }^{*}$ \\ Yuko Doi, ${ }^{\dagger}$ Yohei Yamamoto, ${ }^{*}$ Masayuki Yoshida, ${ }^{*}$ \\ Takuo Tokairin, ${ }^{*}$ Toshiaki Yoshioka, ${ }^{*}$ \\ Yasufumi Omori, ${ }^{*}$ and Katsuhiko Enomoto* \\ From the Department of Pathology" and the Central Research \\ Laboratory, ${ }^{\dagger}$ Akita University School of Medicine, Akita, Japan
}

Orthovanadate (OV), an inhibitor of protein tyrosine phosphatases, affects various biological processes in a cell-type-specific manner. In this study, we investigated the effect of OV on hepatic stellate cells (HSCs). When primary rat HSCs were cultured in the presence of $10 \%$ serum, they spontaneously lost characteristic stellate morphology, proliferated, and were transformed into an activated state with the formation of abundant stress fibers and increased expression of both $\alpha$-smooth muscle actin and collagen type I mRNA. OV treatment inhibited proliferation and activation of HSCs and partially reversed the phenotype of activated HSCs. Among the signaling molecules investigated, phosphorylation of the Src protein at tyrosine $\mathbf{4 1 6}$ was the most striking in OV-treated HSCs. Treatment of cells with Src family inhibitors partially abrogated the effects of OV. Furthermore, transfection of v-Src into activated HSCs induced a stellate morphology similar to that in the quiescent state. We then examined whether $O V$ could effectively suppress HSC activation in vivo after liver injury induced by either carbon tetrachloride or dimethylnitrosamine. OV significantly reduced the appearance of $\alpha$-smooth muscle actin-positive cells and decreased collagen deposition, concomitant with an improvement in liver function. Our study showed for the first time that OV was able to suppress the activation of HSCs, possibly through the modulation of Src activity, and attenuated fibrosis after chronic liver injury. (Am J Pathol 2009, 174:881-890; DOI: 10.2353/ajpath.2009.080261)

Vanadium is an ultratrace element in vertebrates and its total body store is estimated to be $\sim 100 \mu \mathrm{g}$ in humans. ${ }^{1}$ In the biological system, vanadium is found predominantly as vanadate $[+5$; orthovanadate $(\mathrm{OV})$ and meta- vanadate] or vanadyl $(+4)$, with the transition between these forms in the cytoplasm. ${ }^{2}$ Although its essentiality has not been proved in humans, it is considered to have many regulatory roles in the body. Exogenously administered vanadium compounds have been shown to exert various biological effects, most notably, insulin-mimetic actions. ${ }^{3}$

The biological actions of the vanadium compounds are complex and their exact mechanisms remain elusive. However, in the case of OV, which has a phosphate-like structure, the inhibition of various phosphoryl transfer enzymes, including protein tyrosine phosphatases and ATPases, could be the most relevant to the biological effects. ${ }^{1,3}$ In particular, OV has been shown to bind to the active center of protein tyrosine phosphatases,${ }^{4}$ thereby strongly inhibiting their activity and inducing a prolonged state of increased protein tyrosine phosphorylation of cellular proteins. ${ }^{5,6}$ At least part of the insulin-mimetic action is attributable to inhibition of protein tyrosine phosphatases. ${ }^{7}$

We previously demonstrated that OV enhanced branching morphogenesis and proliferation of rat hepatocytes cultured within collagen gels, ${ }^{8}$ and that it prevented apoptotic death of rat endothelial sinusoidal cells. ${ }^{9}$ In long-term cultures of rat hepatocytes, ${ }^{8}$ we noticed that OV almost completely prevented proliferation of contaminated hepatic stellate cells (HSCs) in the hepatocyte fraction. This prompted us to investigate the effect of OV on purified rat HSCs. In the present study, we have shown that OV strongly inhibits proliferation of rat HSCs and liver fibrosis, and proposed the possible mechanisms of the action.

\section{Materials and Methods}

\section{Isolation and Culture of Rat HSCs}

HSCs of aged male F344 rats were isolated by pronasecollagenase perfusion and subsequent purification by a

Supported in part by the Ministry of Education, Science, Sports, and Culture of Japan (grants 16590303 and 18590362).

Accepted for publication December 2, 2008.

Supplemental material for this article can be found on http://ajp. amjpathol.org.

Address reprint requests to Yuji Nishikawa, Department of Pathology, Akita University School of Medicine, 1-1-1 Hondo, Akita 010-8543, Japan. E-mail: nishikwa@med.akita-u.ac.jp. 
single-step Nycodenz gradient. ${ }^{10}$ Isolated HSCs were plated onto collagen-coated plastic dishes and cultured in Williams' E medium supplemented with $10 \%$ fetal bovine serum and $10 \mathrm{mmol} / \mathrm{L}$ nicotinamide. All of the animal experiments adhered to the criteria outlined in the Guide for the Care and Use of Laboratory Animals prepared by the Institute of Laboratory Animal Resources Commission on Life Sciences, National Research Council (National Academy Press, 1996).

To examine the effects of increased protein tyrosine phosphorylation on HSC activation, OV (sodium orthovanadate dissolved in saline; Wako Pure Chemical, Osaka, Japan) was added to the medium at various concentrations 3 hours after plating. OV was also applied to HSCs activated by culturing for 7 to 10 days. The number of cells per well was estimated by Hoechst 33258-based DNA fluorometric assay after 7 days. In some experiments, Src family inhibitors (PP1 and PP2; Calbiochem, San Diego, CA) were applied.

\section{Western Blot Analysis}

Cells were homogenized in a lysis buffer (1\% Triton $\mathrm{X}-100,1 \%$ sodium deoxycholate, $0.1 \%$ sodium dodecyl sulfate, $158 \mathrm{mmol} / \mathrm{L}$ sodium chloride, in $10 \mathrm{mmol} / \mathrm{L}$ Tris$\mathrm{HCl}$ buffer, $\mathrm{pH}$ 7.5) with a protease inhibitor cocktail (Sigma, St. Louis, MO), $1 \mathrm{mmol} / \mathrm{L} \mathrm{OV}$, and $50 \mathrm{mmol} / \mathrm{L}$ $\mathrm{NaF}$. Extracted protein samples were electrophoresed in $10 \%$ polyacrylamide gels ( $40 \mu \mathrm{g}$ per lane) and transferred to polyvinylidene difluoride membranes. Primary antibodies used were anti-proliferating cell nuclear antigen (PCNA) (Santa Cruz Biotechnology, Santa Cruz, CA), anti- $\alpha$-smooth muscle actin ( $\alpha$-SMA) (DAKO, Carpinteria, CA), anti-desmin (Sanbio, Uden, The Netherlands), antivimentin (DAKO), and anti-Src [Src, phospho-Src (Tyr416), phospho-Src (Tyr-527); Cell Signaling Technology, Beverly, MA]. Detection was performed with enhanced chemiluminescence reagents (GE Health Care, Buckinghamshire, UK). Equal loading was verified on each blot by probing with vimentin antibody because vimentin expression was found to be fairly constant in cultured HSCs. Unfortunately, freshly isolated HSCs showed only low levels of vimentin expression and thus equal loading could not be visually confirmed by Western blotting. However, protein concentrations were carefully determined using the Bio-Rad protein assay (Bio-Rad Laboratories, Hercules, CA), and the amount and quality of each sample were checked by staining blots with Ponceau S.

\section{Reverse Transcriptase-Polymerase Chain Reaction (RT-PCR)}

Total RNA from cultured HSCs was prepared by using a TRIzol reagent (Invitrogen, Carlsbad, CA) and RT-PCR analysis was performed. A one-step RT-PCR kit (TaKaRa, Ohtsu, Japan) was used for the reaction, in which RNA (0.2 $\mu \mathrm{g} / 20 \mu \mathrm{l}$ reaction) was reverse-transcribed using AMV reverse transcriptase for 30 minutes and then amplified for 25 cycles of 95,60 , and $72^{\circ} \mathrm{C}$ for 30,30 , and 90 seconds, respectively. The specific primers used and the expected fragment sizes were as follows. Collagen type $\mid \alpha($ Coll- $\mid \alpha)$ : forward, 5'-CAAGATGGCATCCCTGGACAG3'; reverse, 5'-AGCTGCACCGACAGCACCATC-3'; 600 bp. Fibronectin: forward, 5'-TGCAATGATCAGGACACCAGG-3'; reverse, 5'-GTAATTCCGGTTGCTGTACAG-3', 600 bp. Plasminogen activator inhibitor I (PAI-I): forward, 5'-CAGCTCCTGCCCTCCGAAAGC-3'; reverse, 5'GTTGAACTTGTTGTTCTGAGCC-3'; 416 bp. Peroxisome proliferator-activated receptor- $\gamma$ (PPAR- $\gamma$ ): forward, 5'AGCATCAGGCTTCCACTATGG-3'; reverse, 5'-GATCGAAACTGGCACCCTTGA-3'; 517 bp. c-Myb: forward, 5'-TGGCAGAAAGT(A/G)CT(A/G)AACCCT-3'; reverse, $5^{\prime}$-TCCAGTGGTTCTTGATAGCA-3'; 313 bp. $^{11}$ GAPDH: forward, 5'-ACCACAGTCCATGCCATCAC-3'; reverse, 5'-TCCACCACCCTGTTGCTGTA-3'; 452 bp.

\section{Immunocytochemistry, F-Actin Staining, and Sirius Red Staining}

Immunocytochemistry for $\alpha$-SMA and desmin was performed on methanol-fixed cultured cells or paraffin sections of formalin-fixed liver tissues. Detection was performed with an LSAB kit (DAKO). F-actin was visualized by rhodamine-phalloidin (Invitrogen). Sirius red staining was used to estimate the extent of liver fibrosis.

\section{Transient Transfection of Cultured HSCs}

To examine the effect of Src activation on the phenotype of HSCs, we performed transient expression experiments. HSCs were activated by culturing for 10 days and co-transfected with Lipofectamine 2000 [4 $\mu \mathrm{l}$ for 35-mm dish (1 $\mathrm{ml}$ of medium); Invitrogen], $1.6 \mu \mathrm{g}$ of the expression vector containing the cDNA encoding wild-type $v$ Src (pc3DNA-v-Src; a gift of Hiroshi Ohnishi, Gunma University, Maebashi, Gunma, Japan) or v-Src with a mutated kinase domain (pc3DNA-v-SrcKM; a gift of Hiroshi Ohnishi), ${ }^{12}$ along with $0.4 \mu \mathrm{g}$ of a reporter construct encoding EGFP (pCAGGS-EGFP; a gift of Jun-ichi Miyazaki, Osaka University, Osaka, Japan). As a control, transfection of pCAGGS-EGFP alone $(2 \mu \mathrm{g})$ was also performed. One day after transfection, morphology of the EGFP-positive cells was observed. In some experiments, localization of activated Src was examined by immunofluorescence microscopy with anti-phospho-Src (Tyr-416) antibody (Cell Signaling Technology) and rhodamine-labeled anti-rabbit immunoglobulin antibody (DAKO).

\section{Induction of Liver Injury and Fibrosis}

In an acute liver injury model, a 1:1 mixture of $\mathrm{CCl}_{4}$ and olive oil (or olive oil for control) was administered by gavage at a dose of $3 \mathrm{ml} / \mathrm{kg}$ body weight. The animals were sacrificed 24,48 , and 72 hours after $\mathrm{CCl}_{4}$ treatment. OV (5 mg/kg) or saline was injected intraperitoneally at 30 minutes before $\mathrm{CCl}_{4}$ treatment. Additional OV or saline injections were performed 24 hours (for animals sacrificed after 48 hours) and 24 and 48 hours (for animals sacrificed after 72 hours) after $\mathrm{CCl}_{4}$ treatment. 
To induce chronic liver injury, $\mathrm{CCl}_{4}$ was administered by gavage at a dose of $1 \mathrm{ml} / \mathrm{kg}$ twice per week for 8 weeks. OV was injected intraperitoneally at the doses of 2,4 , and $8 \mathrm{mg} / \mathrm{kg}$, three times per week. Another chronic liver injury model induced by dimethylnitrosamine (DMN) was also used. DMN (1\% in saline) was intraperitoneally injected at a dose of $1 \mathrm{ml} / \mathrm{kg}$, three times per week, for 5 weeks. OV $(2.5,5$, and $10 \mathrm{mg} / \mathrm{kg})$ was intraperitoneally injected three times per week.

The levels of serum transaminases [alanine aminotransferase (ALT) and aspartate aminotransferase (AST)] and alkaline phosphatase (ALP), creatinine, and urea nitrogen were measured by the DriChem System (Fujifilm, Tokyo, Japan). The extent of fibrosis (Sirius red-stained area) and HSC activation ( $\alpha$-SMA and desmin immunoreactivity) was quantified using morphometry software (KSW-500U; Kurabo, Osaka, Japan).

\section{Hydroxyproline Assay}

In the chronic liver injury models, hepatic collagen content was evaluated by hydroxyproline quantification according to the method described by Edwards and O'Brien ${ }^{13}$ with some modifications. The liver tissues (75 to $100 \mathrm{mg}$ ) were homogenized in $1 \mathrm{ml}$ of phosphate-buffered saline and hydrolyzed in $6 \mathrm{~N} \mathrm{HCl}$ overnight at $120^{\circ} \mathrm{C}$. Five $\mu$ l of hydrolysates were mixed with the same amount of citrate acetate buffer, and then $100 \mu$ l of chloramine T solution was added. After 20 minutes of incubation at room temperature, $100 \mu \mathrm{l}$ of Ehrlich's solution was added.

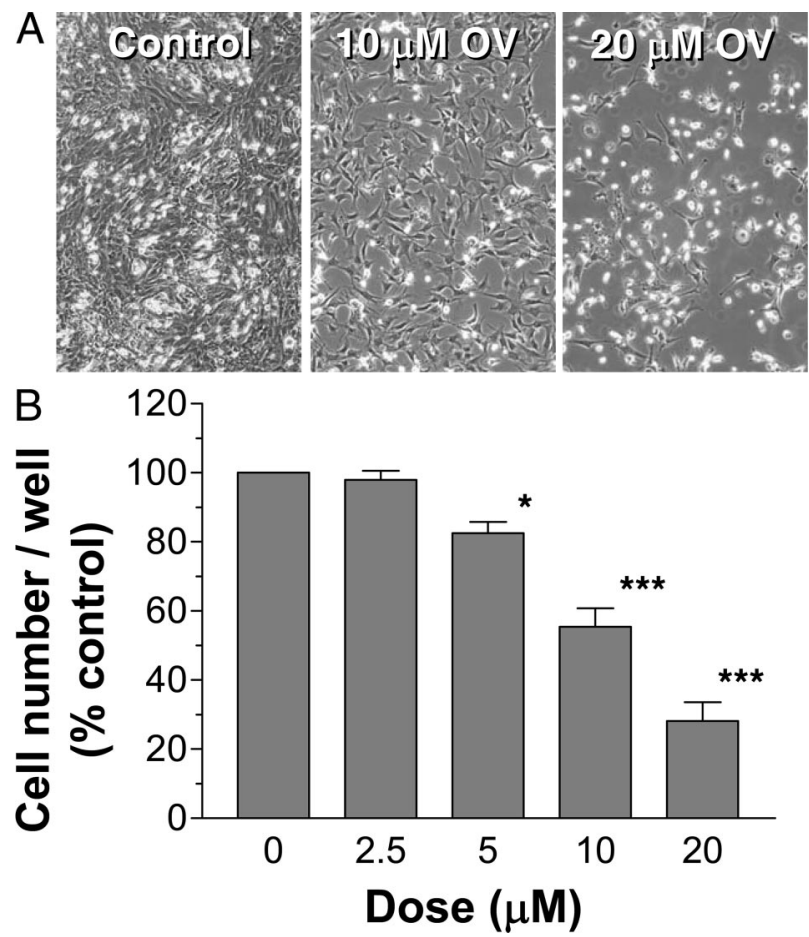

Figure 1. Dose-dependent inhibition of cultured rat HSCs by OV. Cultured for 7 days. A: Phase-contrast micrographs (with a $\times 10$ objective). B: DNA fluorometric quantification of cell number in HSC culture. Each data point is expressed as percentage of control (means \pm SEM for five independent experiments). ${ }^{*} P<0.05$, ${ }^{* * *} P<0.001$; compared with control; one-way analysis of variance.
The mixtures were incubated for 15 minutes at $65^{\circ} \mathrm{C}$ and then absorbance was read at $550 \mathrm{~nm}$. A standard curve was prepared with purified hydroxyproline (Sigma). The hepatic hydroxyproline content was expressed as $\mu \mathrm{g} / \mathrm{g}$ wet liver weight.

\section{Results}

\section{OV Inhibits HSC Proliferation and Activation in Vitro}

After being plated, HSCs spontaneously demonstrated an activated phenotype and began to proliferate (Figure 1A). OV inhibited their proliferation dose dependently (Figure 1, A and B) and maintained the stellate morphology characteristic of quiescent HSCs (Figure 2). Abundant stress fibers were present in the extended cytoplasm of the activated HSCs, but their amount was much lower in the OV-treated cells (Figure 2). OV partially retained the retinol uptake capacity that was lost in activated HSCs (Figure 2).

Expression of $\alpha$-SMA protein, the most reliable marker for HSC activation, became apparent after 7 days in control HSCs, but the expression was almost completely

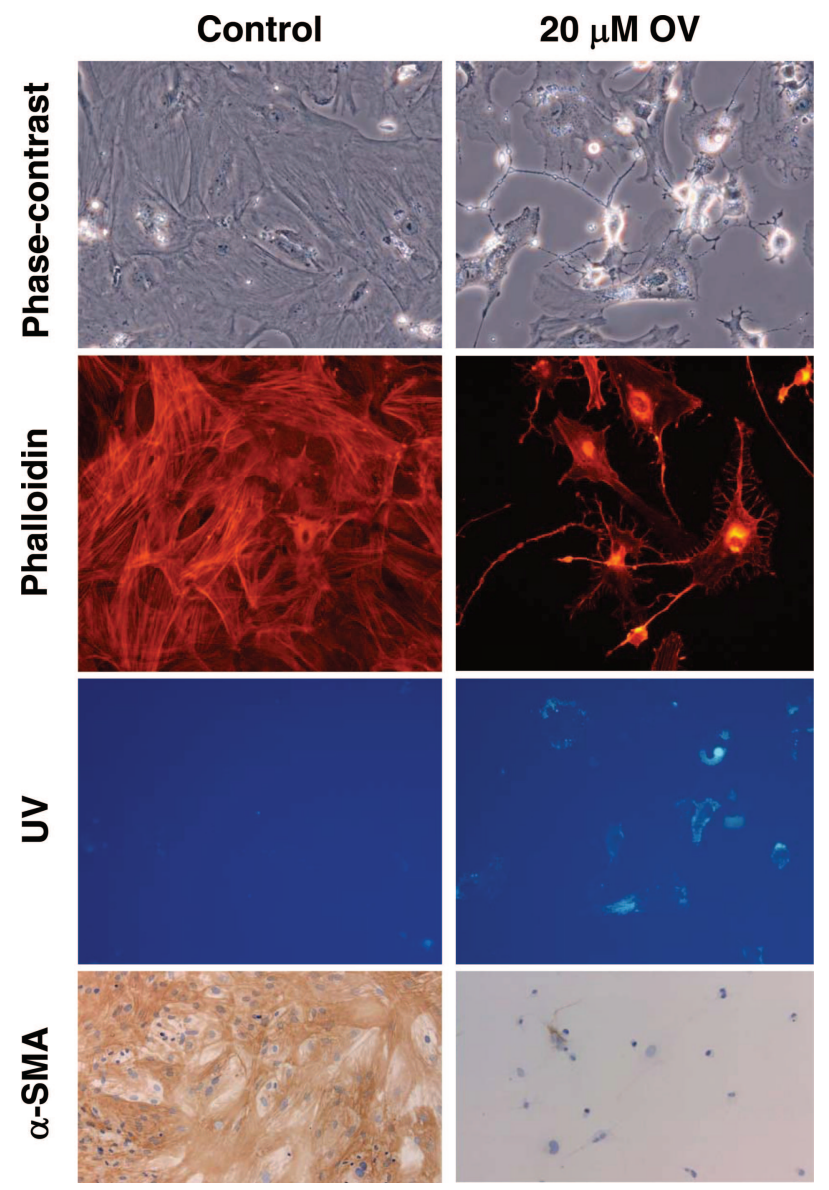

Figure 2. Maintenance of the stellate shape with few stress fibers and the capacity for retinoid uptake in OV-treated HSCs. Cultured for 14 days. From the top, phase-contrast microscopy, rhodamine-phalloidin staining for F-actin, UV autofluorescence of retinoid (cultured in the presence of $10 \mu \mathrm{mol} / \mathrm{L}$ retinol), and $\alpha$-SMA immunocytochemistry (with a $\times 20$ objective). 
A

\begin{tabular}{l|l|l|l|l|} 
O & 0 day & \multicolumn{2}{|l|}{3 days } & 7 days \\
\hline- & - & + & - & + \\
\hline
\end{tabular}
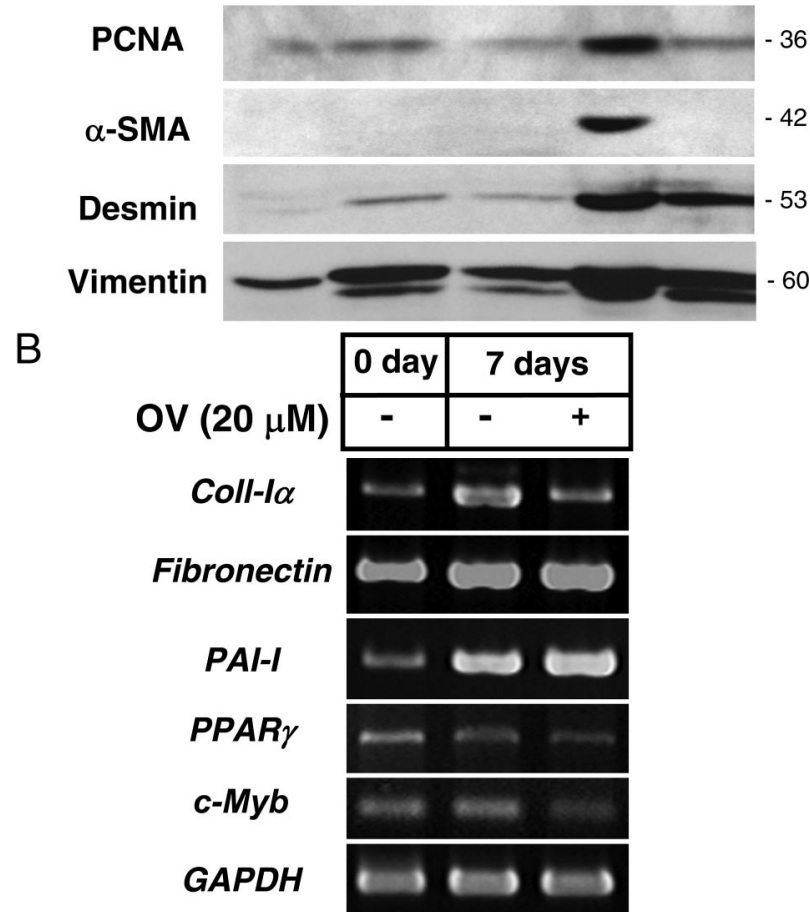

Figure 3. Suppressed expression of $\alpha$-SMA, PCNA, and type I collagen mRNA in cultured HSCs in the presence of OV. A: Western blot analysis of expression of PCNA, $\alpha$-SMA, desmin, and vimentin in freshly isolated HSCs ( 0 day) and cultured HSCs (cultured for 3 days or 7 days). B: RT-PCR analysis of gene expression of collagen type I $\alpha$ (Coll-I $\alpha)$, fibronectin, plasminogen activator inhibitor-I (PAI-I), peroxisome proliferator-activated receptor $\gamma$ (PPAR- $\gamma$ ), c-Myb, and GAPDH.

inhibited in cells treated with OV (Figure 3A). Immunocytochemical analysis of 14-day cultured HSCs also demonstrated the suppressive effect of OV on $\alpha$-SMA expression (Figure 2). Although expression of desmin protein, a marker for both quiescent and activated HSCs, ${ }^{14}$ gradually increased in cultured cells, it was only modestly inhibited by OV (Figure 3A). The expression level of vimentin protein was low in the freshly isolated HSCs, but it increased during culture and was not affected by OV (Figure 3A). As expected from the inhibitory effect on HSC proliferation, OV inhibited expression of PCNA protein (Figure 3A).

In the activated HSCs, gene expression of collagen type $\mid \alpha$, fibronectin, and PAI-I was increased, whereas that of PPAR- $\gamma$ was decreased (Figure 3B). Gene expression of collagen type $\mid \alpha$ was strongly inhibited by OV, although expression of other genes was not affected (Figure 3B). OV also inhibited c-Myb gene expression, which is known to positively regulate differentiation toward smooth muscle cells (Figure 3B). ${ }^{15}$

To examine whether OV could reverse the activated phenotype of HSCs, cells were activated by culturing 7 to 10 days and then cultured for an additional 7 days in the presence of OV. After addition of OV, extended cytoplasm rich in stress fibers gradually shrank and arborizing fine cellular processes emerged, and the HSCs re-
A
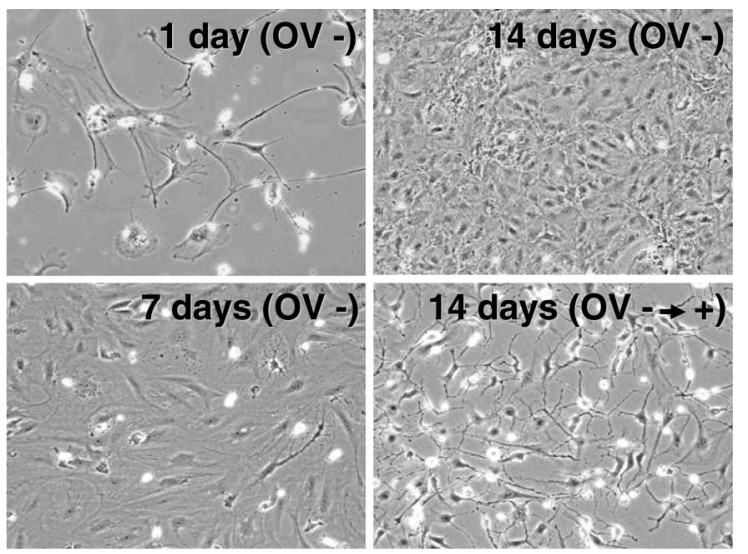

B

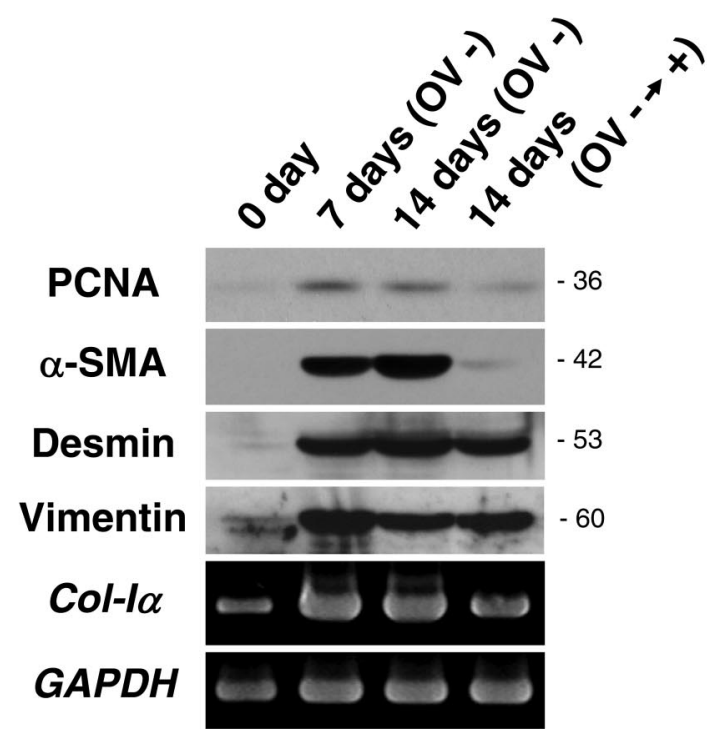

Figure 4. Partial recovery of quiescent phenotype in activated HSCs after OV treatment. HSCs were cultured for 7 days, after which OV $(20 \mu \mathrm{mol} / \mathrm{L})$ was applied (total culturing period, 14 days). A: Phase-contrast micrographs (with a $\times 10$ objective). B: Analysis of expression of PCNA, $\alpha$-SMA, desmin, vimentin (Western blotting), and type I collagen and GAPDH mRNA (RT-PCR).

covered the stellate morphology similar to quiescent cells (Figure 4A). These morphological changes were accompanied by a decrease in the expression of PCNA, $\alpha$-SMA, and collagen type $1 \alpha$ (Figure 4B).

\section{OV Induces Src Phosphorylation at Tyrosine 416 in Cultured HSCs}

To investigate the signaling pathways that might be involved in the action of OV, we performed Western blot analyses using phospho-specific antibodies to several major signaling molecules. OV treatment only marginally enhanced phosphorylation of ERK, Stat3, and Akt, and it did not affect phosphorylation of p38 MAPK, suggesting that its effect on HSCs could not be explained by the modulation of the signaling pathways involving these molecules (data not shown).

We next examined the phosphorylation status of Src, whose activity has been shown to be regulated by phosphorylation of several tyrosine residues. ${ }^{16}$ Although Src was autophosphorylated at tyrosine 416 in the freshly 
A

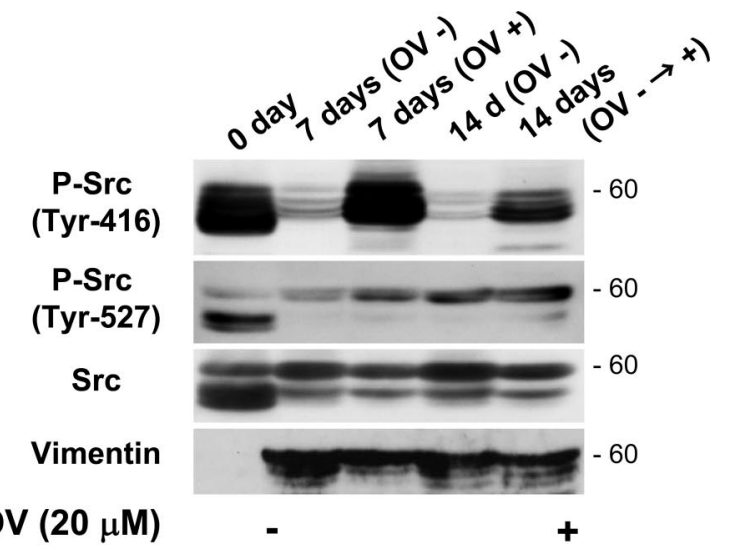

$B \operatorname{OV}(20 \mu \mathrm{M})$
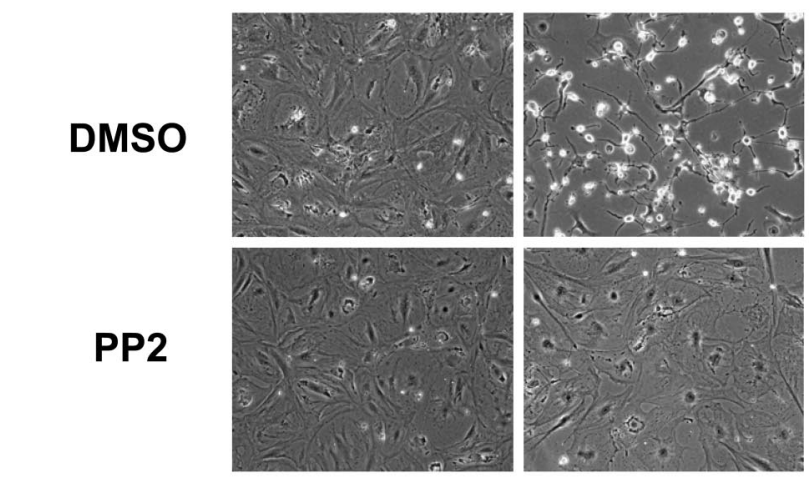

C OV $(20 \mu \mathrm{M})$

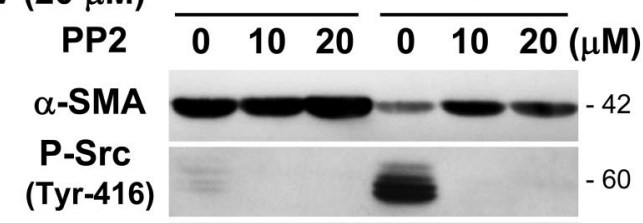

\section{Src}

Vimentin

$-60$

Figure 5. Induction of Src phosphorylation at Tyr- 416 by OV and partial abrogation of OV's effect by a Src-specific inhibitor in cultured HSCs. A: Western blot analysis of tyrosine-phosphorylated Src (Tyr-416 and Tyr-527), total Src, and vimentin. HSCs were cultured for 7 days (lanes 2 and 3) or 14 days (lanes 4 and 5). OV $(20 \mu \mathrm{mol} / \mathrm{L})$ was applied at the start of culture (lane 3) or after 7 days (lane 5). B: Phase-contrast micrographs (with a $\times 10$ objective). HSCs were cultured for 7 days with or without OV in the presence of the Src inhibitor PP2 $(10 \mu \mathrm{mol} / \mathrm{L})$ or vehicle control (dimethyl sulfoxide). C: Western blot analysis of $\alpha$-SMA, phosphorylated Src (Tyr-416), total Src, and vimentin. HSCs were first cultured for 10 days, after which OV and PP2 were added and further cultured for 7 days.

isolated HSCs, it was markedly dephosphorylated during culture (Figure 5A). In the presence of OV, however, tyrosine 416 phosphorylation was maintained (Figure 5A). Src phosphorylation at tyrosine 527 increased during culture, but the extent was small and only mildly enhanced by OV (Figure 5A). The preferential phosphorylation at tyrosine 416 by OV was also observed in different cells, such as a human hepatocellular carcinoma cell line (HuH7) (see Supplemental Figure S1 at http://ajp.amjpathol.org). Both PP1 and PP2 strongly inhibited the OV-induced morphological changes (PP2, Figure 5B; PP1, data not shown). Fur-

thermore, PP2 partially abrogated the inhibition of $\alpha$-SMA expression by OV (Figure 5C).

\section{Transient Transfection of $v$-Src Induces a Stellate Morphology in Activated HSCS}

The above results suggested that Src activation might be associated with the suppressive effect of OV. We then examined whether the introduction of constitutively active Src $(\mathrm{v}-\mathrm{Src})$ affected the phenotype of activated HSCs. When the EGFP expression vector (pCAGGS-EGFP) alone was transfected or the expression vector encoding the kinase-defective v-Src mutant (pc3DNA-v-SrcKM) was co-transfected with pCAGGS-EGFP into activated HSCs, the vast majority of EGFP-positive cells (96\% for pCAGGS-EGFP alone, 89\% for co-transfection with pCAGGS-EGFP and pc3DNA-v-SrcKM) showed a flat polygonal morphology with extended cytoplasm containing stress fibers (Figure 6A, top). In contrast, when activated HSCs were co-transfected with the expression vector encoding wild-type v-Src (pc3DNA-V-Src) and pCAGGS-EGFP, nearly all (96\%) of the EGFPpositive cells demonstrated a stellate shape, with the rounded cell body and several elongated dendritic cell processes, which was similar to that induced by OV (Figure 6A, bottom). Immunofluorescence microscopy revealed that EGFP-positive cells with a stellate morphology contained abundant Src protein that was phosphorylated at tyrosine 416 (Figure 6B).

A
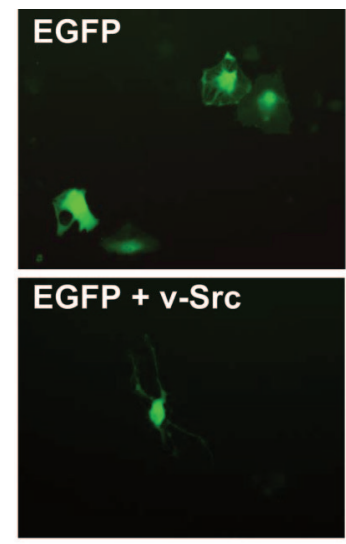

B

\section{EGFP + v-Src}
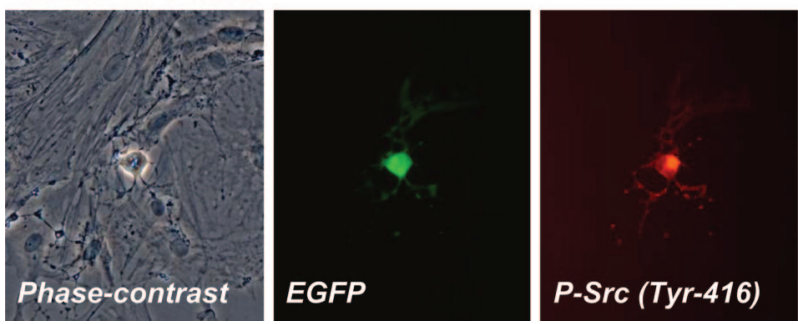

Figure 6. Effect of transient expression of v-Src in activated HSCs. HSCs were activated by culturing for 10 days before lipofection. Photographs were taken 1 day after transfection with a $\times 20$ objective. A: Transfection with vectors for the expression of EGFP, kinase-defective $\mathrm{v}$-Src mutant ( $\mathrm{v}-\mathrm{SrcKM}$ ), and $\mathrm{v}$-Src B: Co-transfection with EGFP and v-Src expression vectors. Phosphorylated $\mathrm{Src}(\mathrm{Tyr}-416)$ is present in an EGFP-positive HSC. 
A

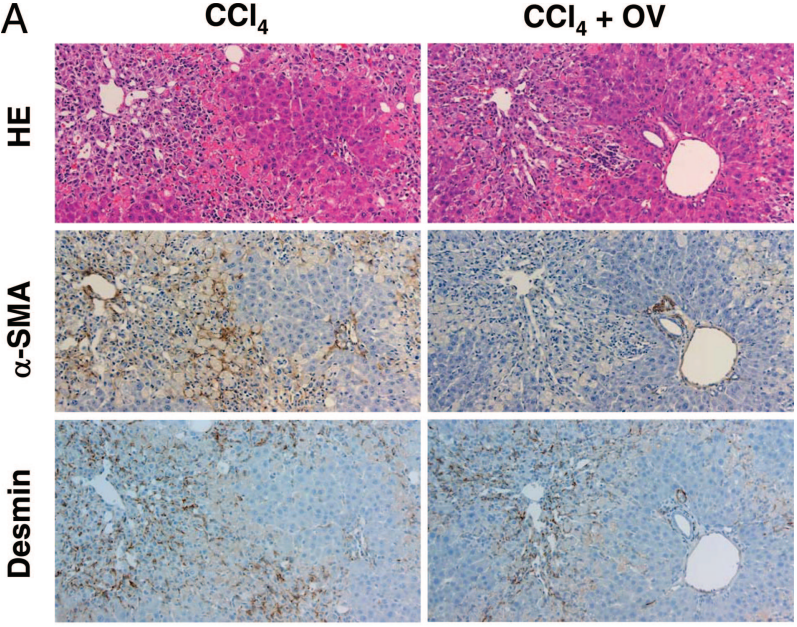

B
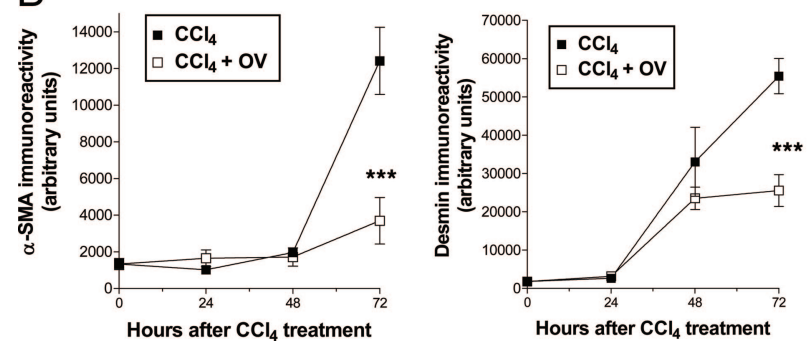

Figure 7. Suppression of HSC activation by systemic administration of OV in an acute $\mathrm{CCl}_{4}$ injury model. Single administration of $\mathrm{CCl}_{4}$. A: $\mathrm{H} \& \mathrm{E}$ staining and $\alpha$-SMA and desmin immunohistochemistry of the liver (with a $\times 10$ objective) after 72 hours. B: Quantitative analysis of $\alpha$-SMA immunoreactivity (left) and desmin-immunoreactivity (right). Each data point represents mean \pm SEM for nine (control) and six $\left(\mathrm{CCl}_{4}\right.$ and $\left.\mathrm{CCl}_{4}+\mathrm{OV}\right)$ rats. ${ }^{\text {*** }} \mathrm{P} P<$ 0.001 as compared with $\mathrm{CCl}_{4}$ alone at 72 hours; one-way analysis of variance.

\section{OV Inhibits Myofibroblastic Transformation of HSCs in Vivo and Suppresses Liver Fibrosis}

$\mathrm{CCl}_{4}$ treatment caused a marked increase in transaminases and centrilobular necrosis to a similar extent in saline- and OV-treated animals (ALT levels: see Supplemental Figure S2A at http://ajp.amjpathol.org; histology: Figure 7A). The appearance of $\alpha$-SMA-positive HSCs, which occurred after 72 hours in the control, was dramatically suppressed, whereas $\alpha$-SMA expression in vascular smooth muscle cells was maintained in OV-treated animals (Figure 7, A and B). Although OV did not affect the appearance of desmin-positive HSCs in the necrotic area at 2 days, a further increase in desmin-positive cells was inhibited by OV (Figure 7, A and B).

We then examined the effect of $\mathrm{OV}$ in a chronic $\mathrm{CCl}_{4}$ injury model. Chronic OV treatment did not induce any physical symptoms or behavioral alterations, except for a slight decrease in body weight at doses more than $8 \mathrm{mg} / \mathrm{kg}$ (see Supplemental Figure S2B at http://ajp.amjpathol.org). Because it has been reported that OV has potential toxicity, particularly to the kidney, ${ }^{17}$ the serum levels of creatinine and urea nitrogen were measured in animals chronically treated with OV. The level of creatinine increased in a dose-dependent manner, whereas that of urea nitrogen was almost unchanged, suggesting some metabolic perturbation, but not renal failure, occurred in
OV-treated animals (see Supplemental Figure S3 at $h t t p: / /$ ajp.amjpathol.org). Indeed, there were no discernible histological abnormalities in the kidneys (see Supplemental Figure S4 at http://ajp.amjpathol.org). The histology of other major organs, including the liver, pancreas, spleen, heart, and lungs, was also unremarkable (see Supplemental Figure S4 at $h$ ttp://ajp.amjpathol.org).

After repeated $\mathrm{CCl}_{4}$ treatments for 8 weeks, a marked body weight loss occurred, but it was ameliorated by OV treatment (see Supplemental Figure S2B at http://ajp. amjpathol.org). In animals treated with $\mathrm{CCl}_{4}$ only, the liver became shrunken and increased its consistency (Figure $8 \mathrm{~A}$ ), and the serum levels of transaminases (ALT and AST) and ALP were elevated (Figure 8B; AST, data not shown). The elevated levels of these enzymes were attributable to accumulated effects of repeated $\mathrm{CCl}_{4}$ administrations because animals were sacrificed after 4 days after the last $\mathrm{CCl}_{4}$ administration, when transaminase levels should return to near normal after a single administration of $\mathrm{CCl}_{4}$, which was confirmed in a separate experiment (data not shown). However, administration of OV improved the gross appearances of the liver
A
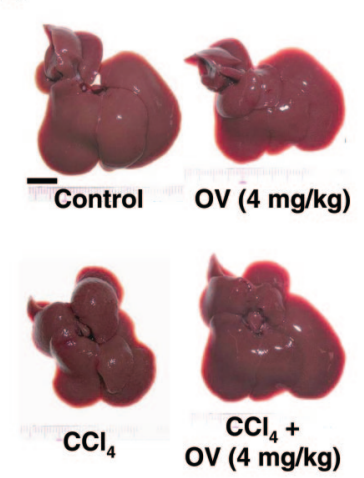

C

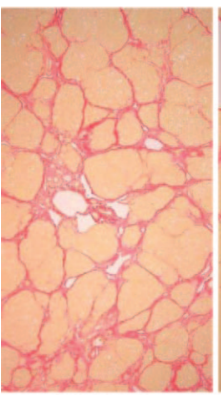

Control

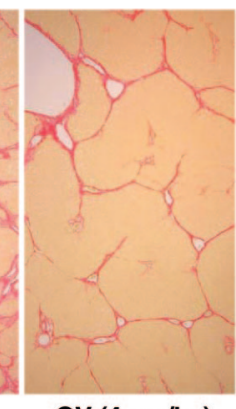

OV (4 mg/kg)
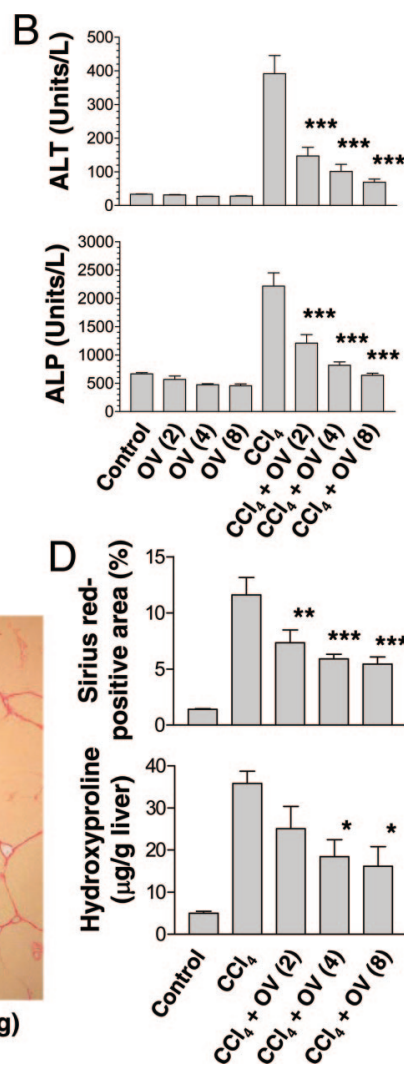

Figure 8. Amelioration of liver injury and suppression of fibrosis by systemic administration of $\mathrm{OV}$ in a chronic $\mathrm{CCl}_{4}$ injury model. Repeated administration of $\mathrm{CCl}_{4}$ for 8 weeks. OV was intraperitoneally injected three times per week at the indicated doses. A: Gross appearances of the livers. B: Serum levels of ALT and ALP. The number in parentheses indicates the dose of OV $(\mathrm{mg} / \mathrm{kg})$. Each data point represents mean \pm SEM for eight rats. ${ }^{* * * *} P<0.001 ; \mathrm{com}$ pared with $\mathrm{CCl}_{4}$ alone; analysis of variance. C: Sirius red staining of the liver (with a $\times 10$ objective). D: Quantitative analysis of Sirius red-positive area and the hydroxyproline content of the liver. The number in parentheses indicates the dose of $\mathrm{OV}(\mathrm{mg} / \mathrm{kg})$. Each data point represents mean $\pm \mathrm{SEM}$ for eight rats. ${ }^{*} P<0.05$; ${ }^{* * *} P<0.01$; ${ }^{* * * *} P<0.001$; compared with $\mathrm{CCl}_{4}$ alone; one-way analysis of variance. Scale bar $=1 \mathrm{~cm}$. 
(Figure $8 \mathrm{~A}$ ) and inhibited the elevation of the transaminases and ALP dose dependently (Figure 8B). Histological analysis revealed that cirrhotic changes with lobular remodeling in the liver of $\mathrm{CCl}_{4}$-treated rats were markedly ameliorated by OV treatment (Figure 8C). This antifibrogenic effect of OV was dose-dependent and statistically significant (Figure 8D). Quantification of hepatic hydroxyproline content confirmed the effect of OV treatment (Figure 8D).

The effect of OV was further examined in a chronic DMN model. The livers in DMN-treated animals were shrunken with dark discoloration because of congestion, whereas OV treatment decreased these gross abnormalities (Figure 9A). DMN treatment elevated the levels of transaminases and ALP, but they were significantly decreased by OV treatment (Figure 9B). As in the chronic $\mathrm{CCl}_{4}$ injury model, the elevated levels of these enzymes were attributable to accumulated effects of DMN administrations, since animals were sacrificed after 5 days after the last DMN administration, when transaminase levels usually returned to near normal after a single administration of DMN. Although chronic DMN treatment increased delicate collagen fibers and $\alpha$-SMA-positive HSCs along
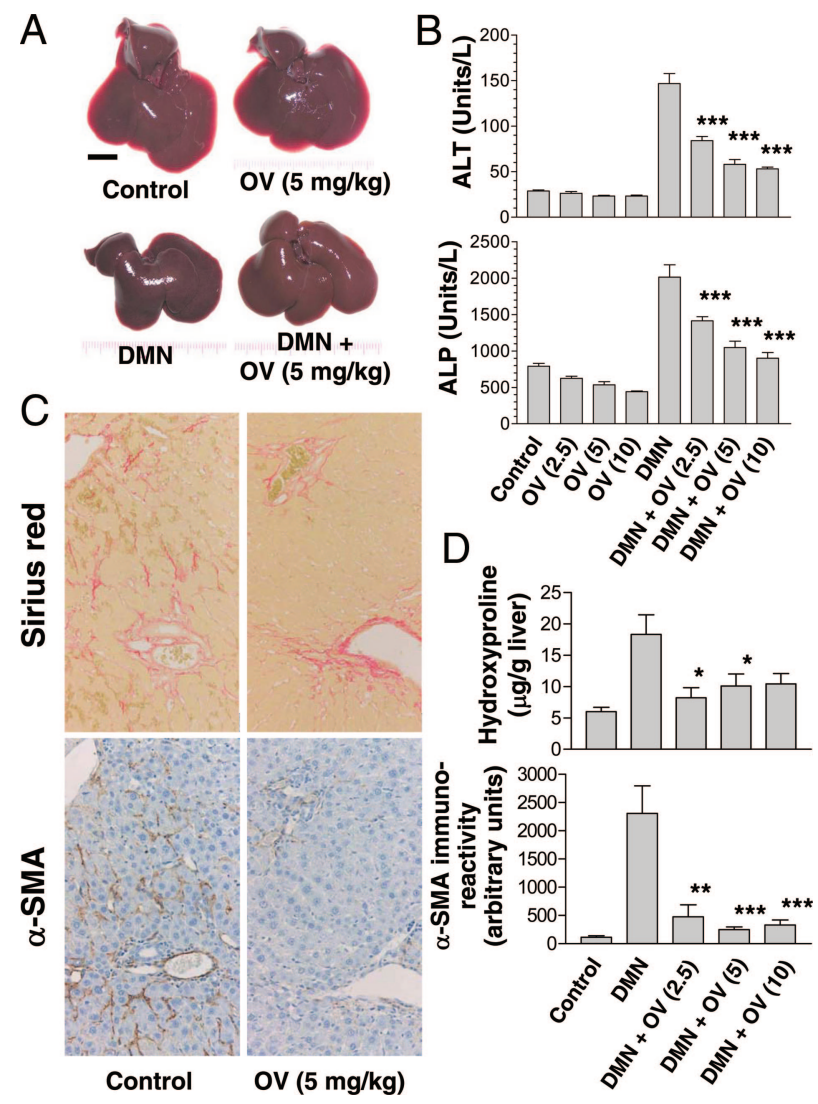

Figure 9. Amelioration of liver injury and suppression of HSC activation in a chronic DMN injury model ( 5 weeks). A: Gross appearances of the livers. B: Serum levels of ALT and ALP (mean \pm SEM) for six rats. The number in parentheses indicates the dose of $\mathrm{OV}(\mathrm{mg} / \mathrm{kg})$. ${ }^{* * * *} P<0.001$; compared with DMN alone; analysis of variance. C: Sirius red staining and $\alpha$-SMA immunohistochemistry of the liver (with a $\times 10$ objective). D: Quantitative analysis of the hydroxyproline content in the liver and $\alpha$-SMA immunoreactivity within the liver lobules (mean \pm SEM) for six rats. The number in parentheses indicates the dose of $\mathrm{OV}(\mathrm{mg} / \mathrm{kg}) .{ }^{*} P<0.05$; ${ }^{* *} P<0.01$; ${ }^{* * * * *} P<0.001$; compared with DMN alone; one-way analysis of variance. Scale bar $=1 \mathrm{~cm}$. the hepatic sinusoids (Figure 9C), OV treatment significantly reduced the extent of collagen deposition, as well as the appearance of $\alpha$-SMA-positive cells (Figure 9, C and D).

\section{Discussion}

In the present study, we showed that OV exerted a strong suppressive effect on HSC activation in vitro and in vivo. One of the interesting features of the biological effect of OV is its prolonged stimulation of tyrosine phosphorylation of many proteins. ${ }^{5,6,18}$ We reported that OV enhanced bile duct-like branching morphogenesis of hepatocytes within collagen gels, which was mainly attributable to stimulation of the MEK-ERK and PI3K-Akt pathways. ${ }^{8}$ We also found that OV suppressed apoptosis of liver sinusoidal endothelial cells through the maintenance of Bad phosphorylation by stimulation of MEK-ERK, p38 MAPK, and PI3K-Akt pathways. ${ }^{9}$ In contrast to the cases of hepatocytes and sinusoidal endothelial cells, OV only modestly affects the phosphorylation status of ERK and Akt, and did not affect that of p38 MAPK in HSCs, indicating that the effect of $\mathrm{OV}$ is cell-type-specific or context-dependent.

Despite the significant inhibitory effects on the expression of $\alpha$-SMA and stress fiber formation, as well as on collagen production and proliferation, OV did not suppress the molecular changes that have been known to closely associate with HSC activation, such as decreased PPAR- $\gamma$ expression ${ }^{19}$ and increased PAI-I expression. ${ }^{20}$ Furthermore, OV rather stimulated phosphorylation of the signaling molecules (ERK, Stat3, and Akt) that have been implicated in the activation of HSCs. ${ }^{21-23}$ Although these established mechanisms for HSC activation may act independently or cooperatively to induce activated phenotype of HSCs, our results suggest that OV might act more directly to the mechanism of actin remodeling with $\alpha$-SMA expression, which is among the most essential characteristics of activated HSCs. The strong effect of OV on $\alpha$-SMA inhibition might also relate to its effect on gene expression of c-Myb that has been demonstrated to positively regulate differentiation to smooth muscle cells. ${ }^{15}$

There was considerable Src phosphorylation at tyrosine 416 in freshly isolated HSCs, but its level was decreased during activation in culture. In contrast, the phosphorylation level of tyrosine 527 increased only modestly during culture. It has been demonstrated that autophosphorylation of tyrosine 416 stimulates Src activity, whereas phosphorylation of tyrosine 527 inhibits it, ${ }^{16}$ rendering its activity dependent on the balance of phosphorylation of these two sites. ${ }^{24}$ Thus, our results suggest that Src activity is high in quiescent HSCs, but decreased when cells are activated. Although there has been a report suggesting that the Src kinase pathway might contribute to HSC activation via inducing thrombospondin-1 and transforming growth factor- $\beta 1,{ }^{25}$ several studies have implicated the possible link between HSC activation and a decrease in Src activity. It has been described that Src kinase activity in cultured human HSCs is high in the quiescent state 
(nonadherent condition), whereas the activity is reduced when activated (adherent condition). ${ }^{26}$ The suppression of Src activity during activation might be mediated by a cell surface glycoprotein (Thy-1), ${ }^{27}$ which increases in myofibroblasts after liver injuries. ${ }^{28}$ Furthermore, there might be a possibility that an increased activity of cell surface transglutaminase, which is associated with HSC activation, induces integrin clustering and suppresses Src activity. ${ }^{24}$

OV induced marked autophosphorylation of Src at tyrosine 416 , whereas it only mildly increased tyrosine 527 phosphorylation, suggesting that OV might maintain or stimulate the activity in HSCs. Our data showing that the effect of OV on HSCs was partly abrogated by the Src family inhibitors support the importance of Src activation in the action of OV. Although incubation of fibroblastic cells with OV has been shown to inhibit Src activity by increasing tyrosine 527 phosphorylation, ${ }^{29}$ the apparently contradictory results may be attributable to shortterm incubation in the study, since the effect of OV on protein tyrosine phosphorylation in viable cells in culture proceeds slowly and steadily for more than 6 hours $^{18}$ and continues for at least several days. ${ }^{8,9}$ Alternatively, because it has been shown that distinct protein tyrosine phosphatases regulate the phosphorylation status of these two tyrosine residues, ${ }^{16}$ the preferential effect on tyrosine phosphorylation may be attributable to a specific protein tyrosine phosphatase expression profile in HSCs suggesting that Src was activated in the presence of OV.

Forced expression of active Src ( $v-S r c)$ in the fully activated HSCs induced a stellate morphology with loss of stress fibers similar to that seen in the quiescent state. Although the mechanism by which Src regulates the phenotype of HSCs is not clear, the morphological change suggests that there might be a reorganization of the actin cytoskeleton. Interestingly, in a mesangial cell line, transfection of $v$-Src has been reported to induce marked suppression of $\alpha$-SMA expression, which is associated with decreased activity of the serum response element (SRE) and the $\alpha$-SMA promoter. ${ }^{30}$ Furthermore, isoproterenol-induced actin disassembly in smooth muscle cells has been demonstrated to be partly mediated by Src activation. ${ }^{31} \mathrm{Src}$ is known to regulate the function of Rho protein, which plays important roles in actin remodeling and myogenic differentiation of HSCs. ${ }^{32,33}$ It has been reported that $\mathrm{Src}$ phosphorylates and activates one of the substrates, p190 RhoGAP, enhancing the conversion of Rho-GTP to Rho-GDP and blocking stress fiber formation. ${ }^{24,34,35}$ Importantly, Brandt and colleagues $^{36}$ have demonstrated that, in a vascular smooth muscle cell line, actin disassembly induced by protein kinase $\mathrm{C}$ is dependent on Src activation and Rho inactivation, which is associated with increased p190RhoGAP phosphorylation. Because the activity of $\mathrm{p} 190$ RhoGAP is negatively regulated by a protein tyrosine phosphatase containing Src homology 2 (SHP2), ${ }^{37}$ it might also be possible that OV enhances the activity of $p 190$ RhoGAP through SHP2 inhibition. Furthermore, an inhibitor of ROCK ( $p 160-$ Rhoassociated coiled-coil-containing protein kinase) (Y-27632) has been shown to inhibit activation of HSCs both in vitro ${ }^{38}$ and in vivo. ${ }^{39}$ These observations suggest that OV-induced
Src activation might modulate the actin cytoskeleton of HSCs and maintain their quiescent state, partly through the inhibition of the Rho signaling pathway. However, further studies are needed to substantiate this possibility.

In agreement with the in vitro observations of the actions of OV on HSCs, OV showed antifibrotic effects in vivo in liver injuries induced by two different hepatotoxic agents. Although our study suggests that HSCs are the major targets of OV in its antifibrotic effects, the protective effects in vivo could be more complex. It is also possible that OV protects other types of cells in the liver, such as sinusoidal endothelial cells, hepatocytes, and Kupffer cells. In fact, as we previously mentioned, systemic treatment with OV exerted strong anti-apoptotic effects on sinusoidal endothelial cells in an ischemiareperfusion liver injury model. ${ }^{9}$ In the present study, although OV did not apparently reduce hepatocyte injury per se, it lowered the levels of transaminases and alkaline phosphatase in the chronic liver injury models. OV could protect hepatocytes either indirectly through improvement of the hepatic microenvironment or directly through influencing their cellular conditions. It is possible that OV might affect regenerative capacity of hepatocytes, because OV has been shown to release contact inhibition of cultured hepatocytes by inhibiting a protein tyrosine phosphatase (LAR), which is associated with and inactivates c-Met. ${ }^{40}$ Furthermore, OV has been shown to affect Kupffer cell functions by inducing tyrosine phosphorylation of GTPase-activating protein. ${ }^{41}$

In our animal experiments, despite attenuation of myofibroblastic transformation of HSCs by OV treatment, tissue repair was not hampered but rather facilitated, resulting in better gross and microscopic appearances of the liver with fewer biochemical abnormalities. Interestingly, similar observations have been made in tendon repair. ${ }^{42,43}$ In these studies, OV inhibited differentiation of fibroblasts to $\alpha$-SMA-positive myofibroblasts, but normal wound contraction took place with more rapid and orderly organization of collagen fiber bundles. Although activation of HSCs could be a prerequisite for tissue repair after liver injuries, excessive myofibroblastic activation of HSCs may be harmful, resulting in liver fibrosis and cirrhosis. ${ }^{44}$

Although vanadium compounds are normally present in animals including humans and the essentiality has been proved in lower species, ${ }^{45}$ there have been several reports showing that vanadium compounds may exert harmful effects, such as nephrotoxicity, when administered in excess. ${ }^{17}$ It has also been shown that OV transiently stimulates proliferation of cultured human mesangial cells. ${ }^{46}$ In our study, we found that chronic administration of OV induced a slight decrease in body weight at higher doses and a dose-dependent increase in the serum level of creatinine. However, OV rather ameliorated the body weight loss in animals with chronic $\mathrm{CCl}_{4}$ injury. Although the mechanism of the increase in serum creatinine in OV-treated animals is currently unclear, it is unlikely to be attributable to renal insufficiency, because it was not associated with an increase of blood urea nitrogen. The examination of major organs in chronically OV-treated animals revealed no evidence of pathological changes, including mesangial prolif- 
eration in the kidney. Because it has been shown that the biological effect of OV is augmented by interaction with hydrogen peroxide by forming peroxivanadate, ${ }^{47}$ hydrogen peroxide locally produced in the injured and inflamed $\operatorname{sites}^{48}$ might enable $\mathrm{OV}$ to act at relatively low dosages without causing systemic adverse effects. It has been demonstrated that various organically chelated vanadium compounds are more potent in its insulin-mimetic action and less toxic. ${ }^{3}$ Further studies are required to examine whether such compounds might be beneficial in suppression of HSC activation and liver fibrosis.

In conclusion, the present study has demonstrated for the first time that OV suppressed HSC activation and liver fibrosis, and suggests that the underlying mechanisms may include Src activation. These findings, together with our previous observations on protective effects on ischemia-reperfusion liver injuries, ${ }^{9}$ suggest that OV or other vanadium compounds might offer therapeutic potential in liver diseases. Our results also highlight the potential importance of regulating the activities of Src kinase in the suppression of HSC activation, which might be a clue to develop new therapeutic strategies.

\section{Acknowledgments}

We thank Dr. Hiroshi Ohnishi (Laboratory of Biosignal Sciences, Institute for Molecular and Cellular Regulation, Gunma University, Maebashi, Japan) for donating pc3DNA-v-Src and pc3DNA-v-SrcKM; Dr. Jun-ichi Miyazaki (Division of Stem Cell Regulation Research, Osaka University Graduate School of Medicine, Osaka, Japan) for pCAGGS-EGFP; and Ms. Eriko Kumagai, Reiko Ito, and Makiko Kawamata for excellent technical assistance.

\section{References}

1. Verma S, Cam MC, McNeill JH: Nutritional factors that can favorably influence the glucose/insulin system: vanadium. J Am Coll Nutr 1998, $17: 11-18$

2. Li J, Elberg G, Crans DC, Shechter Y: Evidence for the distinct vanadyl(+4)-dependent activating system for manifesting insulin-like effects. Biochemistry 1996, 35:8314-8318

3. Thompson $\mathrm{KH}$, Orvig C: Vanadium in diabetes: 100 years from phase 0 to phase I. J Inorg Biochem 2006, 100:1925-1935

4. Denu JM, Lohse DL, Vijayalakshmi J, Saper MA, Dixon JE: Visualization of intermediate and transition-state structures in protein-tyrosine phosphatase catalysis. Proc Natl Acad Sci USA 1996, 93:2493-2498

5. Swarup G, Cohen S, Garbers DL: Inhibition of membrane phosphotyrosyl-protein phosphatase activity by vanadate. Biochem Biophys Res Commun 1982, 107:1104-1109

6. Klarlund JK: Transformation of cells by an inhibitor of phosphatases acting on phosphotyrosine in proteins. Cell 1985, 41:707-717

7. Huyer G, Liu S, Kelly J, Moffat J, Payette P, Kennedy B, Tsaprailis G, Gresser MJ, Ramachandran C: Mechanism of inhibition of proteintyrosine phosphatases by vanadate and pervanadate. J Biol Chem 1997, 272:843-851

8. Nishikawa Y, Doi Y, Watanabe H, Tokairin T, Omori Y, Su M, Yoshioka $\mathrm{T}$, Enomoto $\mathrm{K}$ : Transdifferentiation of mature rat hepatocytes into bile duct-like cells in vitro. Am J Pathol 2005, 166:1077-1088

9. Ohi N, Nishikawa Y, Tokairin T, Yamamoto Y, Doi Y, Omori Y, Enomoto $\mathrm{K}$ : Maintenance of Bad phosphorylation prevents apoptosis of rat hepatic sinusoidal endothelial cells in vitro and in vivo. Am J Pathol 2006, 168:1097-1106
10. Schäfer S, Zerbe O, Gressner AM: The synthesis of proteoglycans in fat-storing cells of rat liver. Hepatology 1987, 7:680-687

11. Jarvis TC, Alby LJ, Beaudry AA, Wincott FE, Beigelman L, McSwiggen JA, Usman N, Stinchcomb DT: Inhibition of vascular smooth muscle cell proliferation by ribozymes that cleave c-myb mRNA. RNA 1996, 2:419-428

12. Ohnishi H, Yamamori S, Ono K, Aoyagi K, Kondo S, Takahashi M: A src family tyrosine kinase inhibits neurotransmitter release from neuronal cells. Proc Natl Acad Sci USA 2001, 98:10930-10935

13. Edwards CA, O'Brien WD, Jr.: Modified assay for determination of hydroxyproline in a tissue hydrolyzate. Clin Chim Acta 1980, 104 161-167

14. Yokoi Y, Namihisa T, Matsuzaki K, Miyazaki A, Yamaguchi Y: Distribution of Ito cells in experimental hepatic fibrosis. Liver 1988 , $8: 48-52$

15. Buck M, Kim DJ, Houglum K, Hassanein T, Chojkier M: c-Myb modulates transcription of the alpha-smooth muscle actin gene in activated hepatic stellate cells. Am J Physiol 2000, 278:G321-G328

16. Roskoski R, Jr.: Src kinase regulation by phosphorylation and dephosphorylation. Biochem Biophys Res Commun 2005, 331:1-14

17. al-Bayati MA, Giri SN, Raabe OG, Rosenblatt LS, Shifrine M: Time and dose-response study of the effects of vanadate on rats: morphological and biochemical changes in organs. J Environ Pathol Toxicol Oncol 1989, 9:435-455

18. Nishikawa Y, Wang Z, Kerns J, Wilcox CS, Carr BI: Inhibition of hepatoma cell growth in vitro by arylating and non-arylating $\mathrm{K}$ vitamin analogs. Significance of protein tyrosine phosphatase inhibition. J Biol Chem 1999, 274:34803-34810

19. Miyahara T, Schrum L, Rippe R, Xiong S, Yee HF, Jr., Motomura K, Anania FA, Willson TM, Tsukamoto $\mathrm{H}$ : Peroxisome proliferator-activated receptors and hepatic stellate cell activation. J Biol Chem 2000 , 275:35715-35722

20. Leyland H, Gentry J, Arthur MJ, Benyon RC: The plasminogen-activating system in hepatic stellate cells. Hepatology 1996, 24:1172-1178

21. Marra F, Gentilini A, Pinzani M, Choudhury GG, Parola M, Herbst H Dianzani MU, Laffi G, Abboud HE, Gentilini P: Phosphatidylinositol 3-kinase is required for platelet-derived growth factor's actions on hepatic stellate cells. Gastroenterology 1997, 112:1297-1306

22. Marra F, Arrighi MC, Fazi M, Caligiuri A, Pinzani M, Romanelli RG, Efsen E, Laffi G, Gentilini P: Extracellular signal-regulated kinase activation differentially regulates platelet-derived growth factor's actions in hepatic stellate cells, and is induced by in vivo liver injury in the rat. Hepatology 1999, 30:951-958

23. Cao Q, Mak KM, Ren C, Lieber CS: Leptin stimulates tissue inhibitor of metalloproteinase-1 in human hepatic stellate cells: respective roles of the JAK/STAT and JAK-mediated H2O2-dependant MAPK pathways. J Biol Chem 2004, 279:4292-4304

24. Janiak A, Zemskov EA, Belkin AM: Cell surface transglutaminase promotes RhoA activation via integrin clustering and suppression of the Src-p190RhoGAP signaling pathway. Mol Biol Cell 2006, 17: $1606-1619$

25. Breitkopf K, Sawitza I, Gressner AM: Characterization of intracellular pathways leading to coinduction of thrombospondin-1 and TGF-beta1 expression in rat hepatic stellate cells. Growth Factors 2005 23:77-85

26. Carloni V, Defranco RM, Caligiuri A, Gentilini A, Sciammetta SC, Baldi E, Lottini B, Gentilini P, Pinzani M: Cell adhesion regulates plateletderived growth factor-induced MAP kinase and PI-3 kinase activation in stellate cells. Hepatology 2002, 36:582-591

27. Barker TH, Grenett HE, MacEwen MW, Tilden SG, Fuller GM, Settleman J, Woods A, Murphy-Ullrich J, Hagood JS: Thy-1 regulates fibroblast focal adhesions, cytoskeletal organization and migration through modulation of p190 RhoGAP and Rho GTPase activity. Exp Cell Res 2004, 295:488-496

28. Dudas J, Mansuroglu T, Batusic D, Saile B, Ramadori G: Thy- 1 is an in vivo and in vitro marker of liver myofibroblasts. Cell Tissue Res 2007, 329:503-514

29. Ryder JW, Gordon JA: In vivo effect of sodium orthovanadate on pp60c-src kinase. Mol Cell Biol 1987, 7:1139-1147

30. Ishikawa $Y$, Kitamura M: Unexpected suppression of alpha-smooth muscle actin, the activation marker of mesangial cells, by pp60v-src tyrosine kinase. Biochem Biophys Res Commun 1998, 244:806-811

31. Hirshman CA, Zhu D, Pertel T, Panettieri RA, Emala CW: Isoproterenol 
induces actin depolymerization in human airway smooth muscle cells via activation of an Src kinase and GS. Am J Physiol 2005, 288:L924-L931

32. Yee HF, Jr.: Rho directs activation-associated changes in rat hepatic stellate cell morphology via regulation of the actin cytoskeleton. Hepatology 1998, 28:843-850

33. Kato M, Iwamoto H, Higashi N, Sugimoto R, Uchimura K, Tada S, Sakai H, Nakamuta M, Nawata H: Role of Rho small GTP binding protein in the regulation of actin cytoskeleton in hepatic stellate cells. J Hepatol 1999, 31:91-99

34. Chang JH, Gill S, Settleman J, Parsons SJ: c-Src regulates the simultaneous rearrangement of actin cytoskeleton, p190RhoGAP, and p120RasGAP following epidermal growth factor stimulation. J Cell Biol 1995, 130:355-368

35. Arthur WT, Petch LA, Burridge K: Integrin engagement suppresses RhoA activity via a c-Src-dependent mechanism. Curr Biol 2000, 10:719-722

36. Brandt D, Gimona M, Hillmann M, Haller H, Mischak H: Protein kinase $\mathrm{C}$ induces actin reorganization via a Src- and Rho-dependent pathway. J Biol Chem 2002, 277:20903-20910

37. Kontaridis MI, Eminaga S, Fornaro M, Zito Cl, Sordella R, Settleman J, Bennett AM: SHP-2 positively regulates myogenesis by coupling to the Rho GTPase signaling pathway. Mol Cell Biol 2004, 24:5340-5352

38. Iwamoto H, Nakamuta M, Tada S, Sugimoto R, Enjoji M, Nawata H: A p160ROCK-specific inhibitor, Y-27632, attenuates rat hepatic stellate cell growth. J Hepatol 2000, 32:762-770

39. Murata T, Arii S, Mori A, Imamura M: Therapeutic significance of Y-27632, a Rho-kinase inhibitor, on the established liver fibrosis. J Surg Res 2003, 114:64-71

40. Machide M, Hashigasako A, Matsumoto K, Nakamura T: Contact inhibition of hepatocyte growth regulated by functional association of the c-Met/hepatocyte growth factor receptor and LAR protein-tyrosine phosphatase. J Biol Chem 2006, 281:8765-8772

41. Chao W, Liu H, Olson MS: Effect of orthovanadate on tyrosine phosphorylation of P120 GTPase-activating protein in rat liver macrophages (Kupffer cells). Biochem Biophys Res Commun 1993, 191:55-60

42. Moyer KE, Saba AA, Hauck RM, Ehrlich HP: Systemic vanadate ingestion modulates rat tendon repair. Exp Mol Pathol 2003, 75: 80-88

43. Ehrlich HP, Keefer KA, Myers RL, Passaniti A: Vanadate and the absence of myofibroblasts in wound contraction. Arch Surg 1999, 134:494-501

44. Iredale JP: Models of liver fibrosis: exploring the dynamic nature of inflammation and repair in a solid organ. J Clin Invest 2007, 117: 539-548

45. French RJ, Jones PJ: Role of vanadium in nutrition: metabolism, essentiality and dietary considerations. Life Sci 1993, 52:339-346

46. Wenzel UO, Fouqueray B, Biswas P, Grandaliano G, Choudhury GG, Abboud HE: Activation of mesangial cells by the phosphatase inhibitor vanadate. Potential implications for diabetic nephropathy. J Clin Invest 1995, 95:1244-1252

47. Heffetz D, Bushkin I, Dror R, Zick Y: The insulinomimetic agents $\mathrm{H} 2 \mathrm{O} 2$ and vanadate stimulate protein tyrosine phosphorylation in intact cells. J Biol Chem 1990, 265:2896-2902

48. Jaeschke H: Mechanisms of liver injury. II. Mechanisms of neutrophilinduced liver cell injury during hepatic ischemia-reperfusion and other acute inflammatory conditions. Am J Physiol 2006, 290:G1083-G1088 